\title{
Improved Spectral Density Measurement from Estimated Reflectance Data with Kernel Ridge Regression
}

\author{
Timo Eckhard ${ }^{1}$, Maximilian Klammer ${ }^{2}$, Eva M. Valero ${ }^{1}$, \\ and Javier Hernández-Andrés ${ }^{1}$ \\ 1 Optics Department, University of Granada, Spain \\ 2 Chromasens GmbH, Konstanz, Germany \\ timo.eckhard@gmx.com
}

\begin{abstract}
Density measurement of printed color samples takes an important role in print quality inspection and process control. When multi-spectral imaging systems are considered for surface reflectance measurement, the possibility of calculating spectral print density over the spatial image domain arises. A drawback in using multi-spectral imaging systems is that some spectral reconstruction algorithms can produce estimated reflectances which contain negative values that are physically not meaningful. When spectral density calculations are considered, the results are erroneous and calculations might even fail in the worst case. We demonstrate how this problem can be avoided by using kernel ridge regression with additional link functions to constrain the estimates to positive values.
\end{abstract}

Keywords: multi-spectral imaging, spectral density, kernel regression.

\section{Introduction}

The objective of color reproduction in printing technology is to reproduce colors of a reference object as faithfully as possible. Print quality control is the task of monitoring the printing process in terms of many factors, such as the accuracy of color reproduction, image resolution or the registration of multicolor print layers [1]. An increasing demand in high-fidelity printing motivates the ongoing research in this field of technology, with the ultimate goal of improving color reproduction and the degree of automation of the printing process.

When multicolor printing presses are considered, the application of ink (ie. the ink thickness) on the paper substrate must be monitored and adjusted individually for each printing unit to maintain high printing quality. Traditionally, color control bars with solid ink patches are printed for this purpose on each printed sheet in a spatial location that is afterwards trimmed off or occluded. For the solid ink patches, changes of ink layer thickness can be approximated from density measurements in a certain range, following the Beer-Lambert law. One measure of such is color density, which can be obtained from an optical filter densitometer with optical filters that are typically specific for the inks to be 
measured. The maximal transmittances of the filter peaks are spectrally located at the corresponding surface reflectance minimum (so the absorption maximum).

An alternative approach to the classical optical filter densitometry is to calculate spectral density from surface reflectance measurements, using narrow-band filter functions. This approach allows spectral density measurements for arbitrary colors and is not limited to those colors defined for color filter densitometry. Due to advances of spectral imaging technologies in recent years, spectral densitometry for printing process control is becoming more and more attractive. Clearly, the real advantage of spectral imaging in printing applications is the possibility of accurate color measurement to determine print quality in high spatial resolution. But the data from such devices can also be used to determine spectral density and potentially be applied to print process control [2].

Line-scan multi-spectral imaging systems qualify specifically for in-line print inspection. In such systems, spectral reflectance at each spatial image location is reconstructed from multi-channel image data. Reconstruction accuracy depends heavily on the system design and the spectral estimation algorithm. Specifically for spectral density measurement from estimated reflectance data a problem occurs, if the estimated reflectance is physically not meaningful due to negative values, an issue that is present in many multi-spectral systems.

In this work we evaluate spectral density measurement with a multi-spectral imaging system and a reflectance estimation approach that guarantees positivity and therefore qualifies for density calculations.

\subsection{Spectral Density Measurements}

The International Organization for Standardization (ISO) has published the ISO 5 norm series for densitometric measurements. For color density measurement corresponding to the classical optical filter approach, several filter functions for different types of standard density are defined, matching specific application domains 3]. For printing applications, the norm filters are matched to the process inks (typically C,M,Y).

Spectral density is defined less restrictive as compared with color density, as it corresponds to computing the density for a particular surface using a narrowband filter function such that a maximal density reading is obtained [1]. The filter peak-wavelength is accordingly adjusted to the minimal reflectance value of the measurement surface. Consequently, the measurement can be considered for other than standard process colors.

We compute this moving filter spectral density $D_{m f}$ for the $m \times 1$ column vector of spectral reflectance $\mathbf{r} \subset \mathbb{R}^{m}$, with $m$ being the dimensionality of reflectance data, as

$$
D_{m f}(\mathbf{r})=-\log _{10}\left(\frac{\mathbf{r}^{T} \mathbf{a}_{\lambda_{m i n}}}{\sum_{i=1}^{m} a_{i}}\right),
$$

with $\mathbf{a}_{\lambda_{\text {min }}}=\left(a_{1}, \ldots, a_{m}\right)^{T} \subset \mathbb{R}^{m}$ being a discretized narrow-band Gaussian shaped filter function, with peak-wavelength $\lambda_{\min }$ corresponding to the index of $\mathbf{r}$ with minimal reflectance. 


\subsection{Spectral Reflectance Estimation}

We consider two regression models for spectral reflectance estimation. A linear least-square model (further PI), and a kernel based ridge regression model (further KL). The PI method is described for instance in [4. The more recently published KL method belongs to the class of so-called Reproducing Kernel Hilbert Space (RHKS) regression models [5]. In this work we apply the logarithmic kernel function, for which details about our implementation are described in [6].

The KL method can be expanded to constrain the solution of the estimation to physically meaningful values, which means that the estimated reflectance spectra have to be positive. Heikkinen et al. propose several so-called link functions that can be used with RHKS regression models to impose this constraint on the solution [7]. These function pairs consist of a forward transformation $\tau$ that is applied to the reflectances $\mathbf{r}$ in the model training phase, and a backward transformation $\tau^{-1}$, applied to the estimate in order to obtain the recovered reflectance $\widetilde{\mathbf{r}}$. In this work, we consider the square root (or root function) and logit transformation function pairs, defined in Table 1. There are several other estimation approaches that constrain the solution to positivity, for instance by using constrained quadratic programming [8]. We consider the KL method because of the high spectral and color accuracy in spectral reflectance estimation reported previously 25667 .

Table 1. Square root and logit transformation functions

\begin{tabular}{lllc}
\hline Link function & $\tau(\mathbf{x})$ & $\tau^{-1}(\mathbf{y})$ & data range \\
\hline Square root & $\mathbf{y}=\sqrt{\mathbf{x}}$ & $\mathbf{x}=\mathbf{y}^{2}$ & $\mathbf{y} \in[0,+\infty), \mathbf{x} \in[0,+\infty)$ \\
Logit & $\mathbf{y}=\ln \left(\frac{\mathbf{x}}{1-\mathbf{x}}\right)$ & $\mathbf{x}=\frac{\exp (\mathbf{y})}{1+\exp (\mathbf{y})}$ & $\mathbf{y} \in(-\infty,+\infty), \mathbf{x} \in(0,1)$ \\
\hline
\end{tabular}

\section{Experiments and Results}

Acquisition System: A 12-channel multi-spectral line scan camera of type truePIXA 1 and a LED line illumination of type Corona II-D50 in combination with a linear translation stage were used for acquisition of multi-channel camera response data. The measurement geometry of the camera observation and illumination angle were set to approximate 45/0 geometry. The linear stage was used to translate color samples under the camera and by that scanned the sample surface. More details on the acquisition system are reported in 9 .

The number of spectral channels in most multi-spectral acquisition systems vary between 3 and 12 . In this study, two configurations of the truePIXA system were considered, namely a 12- and a 3-channel configuration. The system responsivities are illustrated in Figure $1 \mathrm{a}$

${ }^{1}$ http://www.chromasens.de/en/truepixa-spectral-camera 
Dataset: The dataset considered in this work consists of 2698 color patches (see Figure 1b), printed on a 7 ink wide gamut inkjet printer (HP Designjet Z3100). Camera responses were acquired with the above mentioned acquisition system, and averaged spatially over an image area of approximately $2 \mathrm{~mm} \times 2 \mathrm{~mm}$ per patch. Reference measurements of spectral reflectance of each patch were obtained with an ISO 13655 norm conform X-Rite i1iSis XL spectrophotometer 2 .

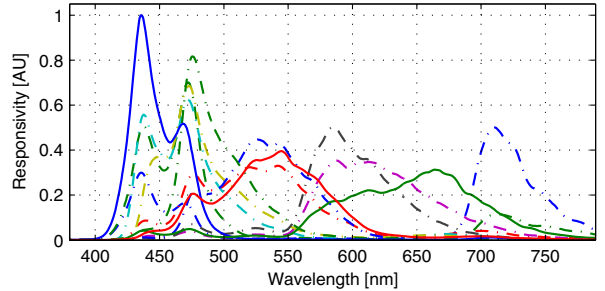

(a)

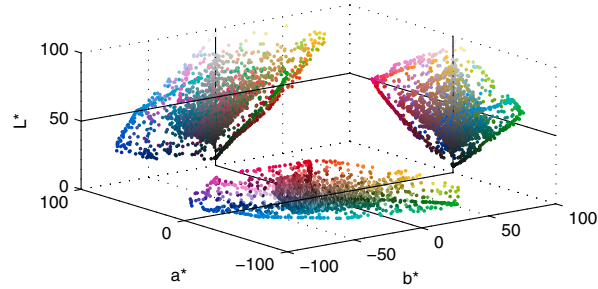

(b)

Fig. 1. a) Spectral responsivity of the imaging system. The solid lines correspond to the 3-channel configuration, dashed-lines and solid lines together correspond to the 12-configuration system; b) CIE-L*a* $\mathrm{b}^{*}$ color coordinates of the 2698 printed color samples, projected onto the coordinate planes.

Evaluation: As outlined in the introduction, the spectral density measurement considered here is based on estimated spectral reflectance data and measurement performance is therefore directly linked to the spectral reflectance estimation performance. We therefore evaluate spectral density measurement performance as well as estimation performance.

The quality of spectral density measurement is evaluated by means of the root mean square error (further RMSE) between reference density $D_{m f}(\mathbf{r})$, calculated from measured reflectances $\mathbf{r}$, and the spectral density $D_{m f}(\widetilde{\mathbf{r}})$, calculated from the corresponding estimated reflectance $\widetilde{\mathbf{r}}$.

We assess the estimation performance spectrally by means of RMSE and colorimetrically by computing CIEDE 2000 color difference 10 , (further $\Delta E_{00}$ ) between color coordinates from estimated and measured reflectances. CIE-L*a*b* coordinates were calculated assuming the CIE-1964 $10^{\circ}$ standard observer and CIE-D65 standard illuminant. The white point was set to the perfect reflecting diffuser.

Our evaluation scheme is based on 10-fold cross-validation. The regularization parameter in the KL method was selected to minimize average RMSE estimation error in a 10-fold cross-validation scheme for the training data. The scale parameter of the logarithmic kernel was fixed to 2 , a value that was found to be appropriate in previous studies [6].

2 https://www.xrite.com/product_overview.aspx?id=894 


\subsection{Negativity of Estimated Reflectance Data}

From the 2698 samples considered in this work, 101 estimated reflectances contain negative values when PI estimation and the 12-channel configuration are considered. For the 3-channel case and PI, 193 reflectances with negative components are found. Using KL method without link functions, only the 3-channel configuration results in estimates with negative values ( 5 samples). One might conclude that using the KL approach with the 12-channel system without any link function could be sufficient for the spectral density measurement task, however, non-negativity is not guaranteed in general.

In Figure 2, we illustrate the color coordinates of measured reflectances that resulted in negative estimates for the 12-channel configuration and PI method. It can be seen that those colors lie mostly close to the gamut boundary, corresponding to highly saturated colors. The spectral density values computed from the measured reflectances indicate that more than $90 \%$ of these samples have spectral densities larger than 2 , with 1.8 corresponding to the sample of minimal spectral density.
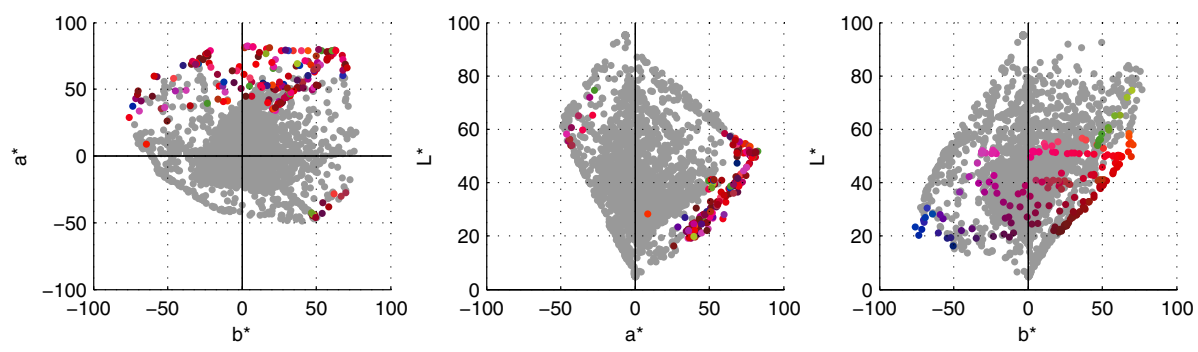

Fig. 2. CIE-L*a*b* color coordinates of the dataset. In color illustrated are reflectances for which the 12-channel configuration and PI method result in negative values.

\subsection{Spectral Estimation Performance}

The numerical results of this evaluation are illustrated in Table 2. For the 12channel configuration and comparison of mean estimation performance, it was found that KL without link function outperforms PI by roughly a factor of 2 in terms of colorimetric and spectral error. Comparing KL results including link functions, it can be seen that no link functions results in the lowest colorimetric error and similar spectral error as for KL with square root link function. Logit link function performance is spectrally worse and in terms of $\Delta E_{00}$ close to that of PI. Comparing maximum colorimetric error values, the logit link function performance is even worse than that of PI, whereas best results are achieved with KL and square root link function. For maximum spectral error, the PI method is almost by a factor of 6 better than any KL approach, with or without link function. 
In case of the 3-channel system, comparison of estimation quality without consideration of link functions shows that PI results on average in a considerable higher spectral and colorimetric error as compared with KL. The maximum spectral error is lowest for PI, but in case of colorimetric error, PI is outperformed by KL. Introducing link functions can reduce the mean estimation performance, this is similar to what was found for the 12-channel case. For logit link function, the performance is worst. However, both link functions provide on average lower errors than the PI method. The overall lowest maximum colorimetric error is achieved with the square root link function. Lowest maximal RMSE is found for PI. Highest maximum colorimetric and spectral errors are found for the logit link function.

Table 2. Reflectance estimation errors for 3- and 12-channel configuration

\begin{tabular}{ccccccccccc}
\hline $\begin{array}{c}\text { Est. } \\
\text { method }\end{array}$ & $\begin{array}{c}\#- \\
\text { chan. }\end{array}$ & $\begin{array}{l}\text { Link } \\
\text { fctn. }\end{array}$ & $\begin{array}{c}\Delta E_{00} \\
\text { Mean }\end{array}$ & Std. & Min. & Max. & Mean & Std. & Min. & Max. \\
\hline \multirow{3}{*}{ KL } & & - & $\mathbf{0 . 2 1}$ & $\mathbf{0 . 1 7}$ & $\mathbf{0 . 0 1}$ & 2.46 & $\mathbf{0 . 0 0 1 8}$ & 0.0024 & $\mathbf{0 . 0 0 0 2}$ & 0.0654 \\
& 12 & logit & 0.38 & 0.38 & 0.02 & 5.44 & 0.0023 & 0.0030 & $\mathbf{0 . 0 0 0 2}$ & 0.0612 \\
& & root & 0.25 & 0.24 & $\mathbf{0 . 0 1}$ & $\mathbf{2 . 4 0}$ & $\mathbf{0 . 0 0 1 8}$ & $\mathbf{0 . 0 0 2 3}$ & $\mathbf{0 . 0 0 0 2}$ & 0.0637 \\
\hline PI & 12 & - & 0.42 & 0.30 & 0.03 & 3.18 & 0.0038 & 0.0024 & 0.0007 & $\mathbf{0 . 0 1 6 2}$ \\
\hline \hline & & - & $\mathbf{0 . 4 1}$ & $\mathbf{0 . 3 1}$ & $\mathbf{0 . 0 1}$ & 4.50 & $\mathbf{0 . 0 0 2 7}$ & $\mathbf{0 . 0 0 4 0}$ & 0.0004 & 0.1265 \\
KL & 3 & logit & 0.63 & 0.71 & $\mathbf{0 . 0 1}$ & 27.75 & 0.0035 & 0.0065 & $\mathbf{0 . 0 0 0 2}$ & 0.2499 \\
& & root & 0.46 & 0.36 & $\mathbf{0 . 0 1}$ & $\mathbf{4 . 0 3}$ & 0.0028 & $\mathbf{0 . 0 0 4 0}$ & 0.0003 & 0.1370 \\
\hline PI & 3 & - & 3.41 & 1.72 & 0.15 & 8.10 & 0.0161 & 0.0146 & 0.0005 & $\mathbf{0 . 0 7 0 9}$ \\
\hline
\end{tabular}

\subsection{Spectral Density Measurement Performance}

Clearly, due to the negativity of estimated reflectances for PI with the 3- and 12channel system configuration, and KL with the 3-channel configuration, we can not evaluate the spectral density measurement performance for all samples in all conditions. The KL method with link functions is therefore the only choice for density measurement of arbitrary datasets. However, by excluding reflectances with negative components from the analysis, a numerical comparison of spectral density measurement performance can still be achieved. Therefore, we provide two sets of results in Table 3 , the left part corresponds to spectral density measurements of the reduced set, and the right part to the results for the full set.

Comparing PI and KL for both, 3 and 12-channel configuration and the reduced set, we see clearly better average performance for the KL approach. For the 12-channel configuration, the lowest average error is found for KL without link function and square root link function, logit performance is only slightly worse. The lowest maximum error is found for the logit function, 2nd lowest for PI, then KL with square root function and KL without link function results in the largest error. For the 3-channel configuration, average RMSE is lowest in 
case of logit and square root function and only slightly worse for KL without link function. The lowest maximum error is again found for the logit function, but unlike in the 12-channel case, the maximum error of PI is larger than that of KL without or with square root link function. The 12-channel configuration performs on average better than the 3-channel system, a finding that is similar to the spectral estimation performance results described in Section 2.2 .

Analyses of spectral density measurement performance based on the full dataset seems to generalize well the reduced dataset case, as can be seen from the comparison of the left and right part in Table 3 .

Table 3. Spectral density measurement quality for the 3- and 12-channel configuration

\begin{tabular}{ccccccccccc}
\hline \multirow{2}{*}{$\begin{array}{c}\text { Est. } \\
\text { method }\end{array}$} & \#- & Link & \multicolumn{1}{c}{ RMSE } & \multicolumn{1}{c}{ (reduced set) } & \multicolumn{5}{c}{ RMSE (full set) } \\
& & fctn. & Mean & Std. & Min. & Max. & Mean & Std. & Min. & Max. \\
\hline & 12 & no & $\mathbf{0 . 0 1 7}$ & 0.062 & $\mathbf{0}$ & 1.345 & 0.018 & 0.061 & $\mathbf{0}$ & 1.345 \\
KL & 12 & logit & 0.018 & $\mathbf{0 . 0 5 8}$ & $\mathbf{0}$ & $\mathbf{1 . 2 6 6}$ & 0.018 & $\mathbf{0 . 0 5 7}$ & $\mathbf{0}$ & $\mathbf{1 . 2 6 6}$ \\
& 12 & root & $\mathbf{0 . 0 1 7}$ & 0.060 & $\mathbf{0}$ & 1.316 & $\mathbf{0 . 0 1 7}$ & 0.059 & $\mathbf{0}$ & 1.316 \\
\hline \multirow{2}{*}{ PI } & 12 & - & 0.057 & 0.113 & $\mathbf{0}$ & 1.271 & - & - & - & - \\
\hline \hline & 3 & no & 0.022 & 0.066 & $\mathbf{0}$ & 1.383 & - & - & - & - \\
KL & 3 & logit & $\mathbf{0 . 0 2 0}$ & $\mathbf{0 . 0 6 0}$ & $\mathbf{0}$ & $\mathbf{1 . 2 9 9}$ & $\mathbf{0 . 0 2 0}$ & $\mathbf{0 . 0 5 8}$ & $\mathbf{0}$ & $\mathbf{1 . 2 9 9}$ \\
& 3 & root & $\mathbf{0 . 0 2 0}$ & 0.062 & $\mathbf{0}$ & 1.349 & 0.021 & 0.061 & $\mathbf{0}$ & 1.349 \\
\hline PI & 3 & - & 0.083 & 0.129 & $\mathbf{0}$ & 1.385 & - & - & - & - \\
\hline
\end{tabular}

\section{Discussion and Conclusions}

We have evaluated spectral density measurement from estimated reflectances of a multi-spectral imaging system in 12 and 3-channel configuration. The calculation of spectral density requires positivity of the sample reflectances. Negative reflectances are physically not meaningful, yet especially the widely used linear least-square regression estimation can result in negative estimates if not avoided by additional constraints. We showed that by constraining the estimation of reflectances using kernel ridge regression and additional link functions, density measurement with estimated reflectance data becomes feasible.

In this work, we have compared linear least-square regression and kernel ridge regression with the logarithmic kernel without and with link functions (logit and square root).

In conclusion, it was identified that kernel ridge regression with link function increases the spectral density measurement performance significantly as compared with linear least-square regression. Constraining the estimates to positivity via usage of a link function in kernel ridge regression reduces the average colorimetric and spectral estimation performance slightly as compared with not using a link function. However, spectral density measurement performance was found to be similar or even higher when using a link function and, what is more important, the estimation produces physically meaningful reflectances. 
Acknowledgements. The authors thank Christoph Godau from the Institute of Printing Science and Technology, Technische Universität Darmstadt for providing the printed color samples and spectral measurements used in this study. This study was supported by Chromasens GmbH through UGR grant number 2936 and by the Spanish Ministry of Research and Innovation through grand number DPI2011-23202.

\section{References}

1. Kipphan, H.: Handbook of print media: Technologies and production methods. Springer (2001)

2. Verikas, A., Bacauskiene, M.: Estimating ink density from colour camera RGB values by the local kernel ridge regression. Eng. Appl. of Artificial Intelligence 21(1), 35-42 (2008)

3. ISO 5:2009 Photography and graphic technology - Density measurements, Part 3: Spectral conditions

4. Ribés, A., Schmitt, F.: Linear inverse problems in imaging. IEEE Signal Processing Magazine 25(4), 84-99 (2008)

5. Heikkinen, V., Lenz, R., Jetsu, T., Parkkinen, J., Hauta-Kasari, M., Jääskeläinen, T.: Evaluation and unification of some methods for estimating reflectance spectra from RGB images. J. Opt. Soc. Am. A 25(10), 2444-2458 (2008)

6. Eckhard, T., Valero, E., Hernndez-Andrs, J., Heikkinen, V.: Evaluating logarithmic kernel for spectral reflectance estimation - effects on model parametrization, training set size and number of sensor spectral channels. J. Opt. Soc. Am. A 31(3), 541-549 (2014)

7. Heikkinen, V., Mirhashemi, A., Alho, J.: Link functions and Matérn kernel in the estimation of reflectance spectra from RGB responses. J. Opt. Soc. Am. A 30(11), 2444-2454 (2013)

8. Park, J.I., Lee, M.H., Grossberg, M.D., Nayar, S.K.: Multispectral imaging using multiplexed illumination. In: IEEE 11th International Conference on Computer Vision, pp. 1-8. IEEE (2007)

9. Godau, C., Klammer, M., Eckhard, T., Schnitzlein, M., Nowack, D., Frei, B., Urban, P.: Evaluation of a multi-spectral camera system for inline color measurement. In: Annual meeting of the German Colour Group (2013)

10. CIE: Improvement to industrial colour-difference evaluation. Tech. rep., CIE Pub. No. 142-2001 (2001) 